\title{
Serious safety concerns regarding use of hydroxyethyl starch for acute fluid resuscitation
}

T he use of hydroxyethyl starch for acute volume resuscitation in critically ill patients is associated with serious safety concerns, according to the authors of a recent paper published in the Journal of the American Medical Association.

Hydroxyethyl starch is a synthetic colloid commonly used in patients who require fluid resuscitation. Although some studies have suggested that hydroxyethyl starch is associated with an increased risk of renal damage and death, results have been mixed. In addition, a number of studies on the topic from one researcher were retracted in 2011 for failing to acquire ethical approval for research and for fabrication of study data.

Ryan Zarychanski and co-workers therefore performed a systematic review and meta-analysis of trial data published up to October 2012, to investigate the association between use of hydroxyethyl starch and mortality and acute kidney injury. "The clinical use of colloidal starch solutions, including hydroxyethyl starch, has increased despite their higher cost relative to crystalloid solutions, lack of evidence of their clinical superiority, and pervasive safety concerns," say the authors. "Over the years, hydroxyethyl starch products have appeared in several resuscitation guidelines, including those of the US Hospital Consortium and have been advocated as the cornerstone of resuscitation therapy."

Zarychanski et al. used numerous databases to identify randomized controlled trials comparing use of hydroxyethyl starch with use of other intravenous fluids for acute fluid resuscitation. From the citations they identified, the researchers included 38 unique trials in their analyses. The trials included between 12 patients and 7,000 patients and were published between 1982 and 2012.

The researchers used data from 10,880 patients in 35 studies to investigate the primary end point of mortality. They found that the pooled relative risk of mortality for patients who were randomly assigned to receive hydroxyethyl starch was 1.07 (95\% CI 1.00-1.14). As most of the included trials were considered to be of high or unclear risk of bias, the researchers graded the strength of the evidence as low. They then performed further analyses after excluding the seven trials from the investigator whose later work was retracted (the investigations into research misconduct were only performed in trials published from 1999 onwards). Once these trials were excluded, pooled results from the remaining 28 studies showed a significant association between hydroxyethyl starch use and death (relative risk 1.09, 95\% CI 1.02-1.17). Without the inclusion of the seven trials-which showed statistical heterogeneity-the strength of the evidence was graded as moderate, and these trials were excluded from further analyses.

Pooled results from 10 trials reporting on the incidence of renal replacement therapy use in 9,258 patients showed that hydroxyethyl starch administration (versus use of other fluids) was associated with a significantly increased risk of receiving renal replacement therapy (relative risk 1.32, 95\% CI 1.15-1.50). The risk of acute renal failure reported in five trials involving 8,725 patients was significantly greater in those receiving hydroxyethyl starch than in those receiving other solutions (relative risk 1.27, 95\% CI 1.09-1.47). The strength of this evidence was graded as being moderate. Administration of hydroxyethyl starch was also associated with a reduction in urine output, but no differences in lengths of stay in the intensive care unit or in hospital were reported.

"Hydroxyethyl starch solutions are effective volume expanders but are not localized to the circulatory system and are known to deposit in the skin, liver, muscle, spleen, endothelial cells, and

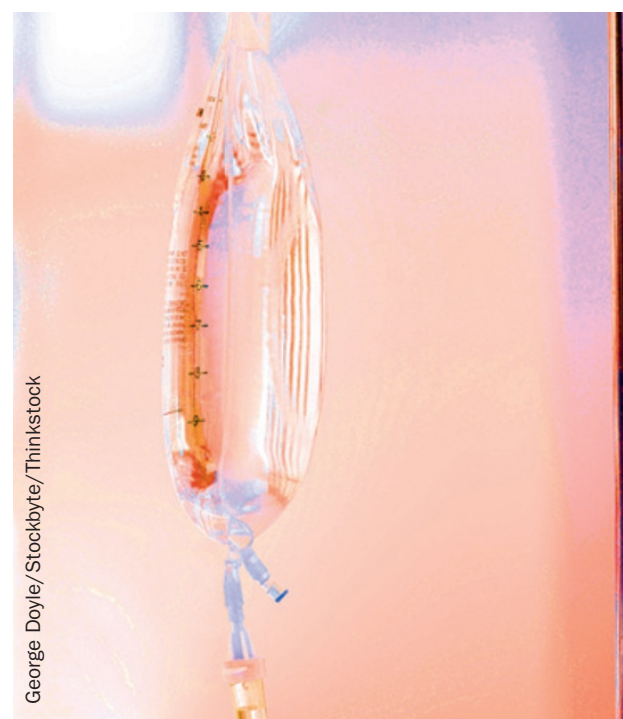

kidneys of patients who receive these products," say the authors. "The toxic effects on renal function have been well documented in experimental and clinical studies, but some researchers have argued that adverse effects depend on the volume and molecular weight of the hydroxyethyl starch preparations and patient population. Proponents of starch solutions have argued increased safety with each newly marketed product, but evidence from randomized trials do not support these claims."

The researchers also state that their systematic review shows how the inclusion of studies with fraudulent data can influence how a body of evidence is interpreted, and how excluding such studies can shift the balance of evidence.

Rebecca Kelsey 